\title{
Polysèmes
}

Revue d'études intertextuelles et intermédiales

\section{Le descriptif à l'encontre de l'image : quelques descriptions tirées de All One Horse. Fictions and Images de Breyten Breytenbach}

\section{Guillaume Cingal}

\section{OpenEdition}

\section{Journals}

Édition électronique

URL : http://journals.openedition.org/polysemes/1742

DOI : 10.4000/polysemes. 1742

ISSN : 2496-4212

Éditeur

SAIT

Édition imprimée

Date de publication : 2 janvier 2007

Pagination : 41-53

ISSN : 0999-4203

\section{Référence électronique}

Guillaume Cingal, «Le descriptif à l'encontre de l'image : quelques descriptions tirées de All One Horse Fictions and Images de Breyten Breytenbach », Polysèmes [En ligne], 9 | 2007, mis en ligne le 01 janvier 2007, consulté le 02 mai 2019. URL : http://journals.openedition.org/polysemes/1742 ; DOI : 10.4000/ polysemes. 1742

Ce document a été généré automatiquement le 2 mai 2019.

Polysèmes 


\title{
Le descriptif à l'encontre de l'image : quelques descriptions tirées de All One Horse. Fictions and Images de Breyten Breytenbach
}

\author{
Guillaume Cingal
}

1 Le poète sud-africain Breyten Breytenbach est aussi connu pour ses activités de peintre. Si l'on se fie même à la diffusion de son œuvre sur Internet (un exemple comme un autre), il semble que le peintre soit plus « médiatisé » que l'écrivain.

Dans All One Horse. Fictions and Images ${ }^{1}$, Breyten Breytenbach livre une alternance de 27 «fictions» (des proses poétiques de deux ou trois pages) et de 27 «images» (des aquarelles). Alors que le sous-titre implique une forte distinction générique (écriture vs. peinture $)^{2}$, le titre est une expression dont l'équivalent français serait «bonnet blanc et blanc bonnet ». Breytenbach s'en explique dans un avant-propos signé « A. Uthor» :

The book is called All One Horse. If you really must know, the title is culled from a Chuang Tzu saying: 'Heaven and earth are one finger, all things are one horse'. This, by the way, also precisely indicates the contents, made up of a structure wrapped in themes or motives. Arguments of course are informed and/or illustrated by imagery or texture, or both. (9)

3 Ce qu'il faut déterminer, par conséquent, outre les éventuelles correspondances thématiques et formelles entre les aquarelles et les textes, c'est si Breytenbach exploite les différences fondamentales entre peinture et écriture, ou s'il cherche à les abolir. Tout semble être une question de méthode : l'approche sémiotique parie plus sur l'interaction que sur le cloisonnement. L'illustration a lieu dans les deux sens, sans primauté de l'une des deux formes d'expression sur l'autre, comme l'indique l'avant-propos. En effet, une des affirmations les plus caractéristiques de Breytenbach est qu'il n'y a pas de différence essentielle entre la poésie et la peinture :

Writing is a continuation of painting just as painting is a prolongation of writing. I

don't indulge in one in the place of the other, or elucidate the one form of 
expression by means of the other. These two disciplines of being share the same means. $^{3}$

Dans Painting the Eye, Breytenbach va même jusqu'à dire que l'interaction entre ces deux formes esthétiques a tout de la métamorphose : la poésie se fait peinture, et inversement ${ }^{4}$.

Une affirmation théorique aussi catégorique, qui a tout pour séduire, mais aussi pour surprendre, passe-t-elle l'épreuve de la mise en pratique? Marilet Sienaert, qui a consacré un article passionnant aux affirmations théoriques de Breytenbach sur l'équivalence des formes esthétiques, s'est surtout attachée à caractériser l'œuvre peint et poétique selon les modalités de l'esthétique de la réception. Elle en a conclu que ce qui primait, c'était la déstabilisation du lecteur ou du spectateur et l'indétermination des signes :

This persistent feature of a shifting $\mathrm{T}$ suggests a general inability to know: there is no reassuring sense of closure, hence the reluctance or even resentment with which one often faces those paintings and poems which forcefully undermine one's conventional perceptions and relatedness to the world. [...] By remaining openended, the poem or painting evokes the right of the individual not to know. ${ }^{5}$

En revanche, Marilet Sienaert ne consacre pas d'analyse à All One Horse, qui est, selon moi, la tentative concrète la plus aboutie et la plus passionnante de Breytenbach, par-delà les affirmations théoriques. C'est bien en artiste que Breytenbach affronte ici le mélange des genres, et qu'il cherche à faire se rencontrer la plume et le pinceau, les mots et les couleurs.

Dans un précédent article, j'ai essayé de montrer que la coïncidence restait en quelque sorte lettre morte (palette sèche?), ou que, tout au moins, Breytenbach s'efforçait de désorienter les affinités de surface entre les aquarelles et les proses poétiques. Il n'y a pas, écrivais-je alors, d'illustration dans All One Horse. La déconstruction n'est pas le seul fait de l'interprète : elle émane bel et bien d'une stratégie d'auteur transformé en bâtisseur de labyrinthe ${ }^{6}$. On pourrait également objecter à A. Uthor, figure d'auteur qui n'est pas l'auteur et manque sans doute d'autorité, que l'expression "all one horse ", quelle que soit sa pertinence philosophique dans le cadre des écrits de Tchouang-Tseu, n'est pourtant pas sans rappeler, en anglais, l'expression idiomatique « a horse of a different colour ", dont le signifié se situe à contre-courant de l'affirmation unitaire d'une interaction de l'expression plastique et de l'expression poétique.

Dans sa dimension essentiellement textuelle, la notion de descriptif postule un paradoxe : des mots peuvent-ils susciter des images? Il s'agit donc de remettre le métier sur l'ouvrage d'un point d'attaque radicalement différent: ce que je nommais naguère la dialectique de la discordance et de la métamorphose prend sans doute une autre tournure à l'aune du « descriptif ».

\section{Magritte, les déictiques et la discrépance}

Avant l'analyse des descriptions de Breyten Breytenbach, un détour.

10 Soit deux tableaux de la période "surréaliste » de Magritte (c'est-à-dire contemporains de l'implication modeste de René Magritte dans le mouvement surréaliste) ${ }^{7}$. L'un s'intitule Table, Océan et Fruit et date de 1927 ; c'est une nature morte représentant une branche d'arbre, une sorte de sac de jute et un pichet; à chacun de ces trois objets se trouve apposée une légende («la table », «l'océan », « le fruit»). Les légendes, que, par 
référence aux écrits d'Isidore Isou sur le théâtre, je qualifierai de "discrépantes " donnent son titre au tableau, mais avec la mise en évidence de l'article défini $(l a, l ', l e)$, à forte valeur déictique.

11 L'autre, de 1930, s'intitule, fort freudiennement, L'Interprétation des rêves. Le même procédé de légendes « discrépantes » y est mis en œuvre : dans chacun des six tableautins d'apparence scolaire se trouve un même décalage entre signe graphique et motif pictural. «L'acacia» est un œuf; «la lune» est une chaussure de dame; "la neige » est un chapeau ; «le plafond » est une bougie ; « l'orage » est un verre ; enfin « le Désert » (avec une majuscule) est un marteau.

12 L'on est tenté de souligner l'influence probable de ces deux tentatives anciennes sur la célèbre série des pipes ; toutefois, le signe graphique n'émet nul paradoxe négatif du style "Ceci n'est pas une pipe ». Au contraire, c'est au spectateur d'établir une syntaxe entre le signe graphique et le motif pictural, comme je viens de le faire dans ma description. Le choix syntaxique est restreint par l'emploi systématique des articles définis, qui différencient aussi ces deux tableaux de la légende «Ceci n'est pas une pipe ».

En termes plus magrittiens, cela pourrait donner: ceci (cet œuf) est l'acacia, ceci (ce soulier) est la lune, etc. Les codes et modes de représentation se trouvent perturbés par ce simple jeu de désignation. En l'occurrence, la "discrépance » insiste sur l'impossibilité d'illustrer une production verbale par des motifs picturaux, et inversement. Dans les deux tableaux cités (et décrits) plus haut, la légende n'est ni négative ni indéfinie; elle est affirmative, dans la mesure où elle postule l'adéquation entre «l'acacia » et l'image de l'œuf.

Dans l'œuvre ultérieure de Magritte, seul le célèbre Effets personnels me paraît en adéquation avec ce modèle : la chambre aux murs bleutés a pour mobilier un lit avec un peigne géant, à gauche; un verre géant au premier plan au centre, avec une allumette géante " allongée » sur le tapis; enfin, un blaireau à raser géant, posé sur l'armoire à droite. Peut-on imaginer, pour ces objets usuels mais déformés par leurs proportions inhabituelles, des légendes discrépantes ? Peut-on élaborer une syntaxe?

\section{L'impossible légende}

Les aquarelles, toutes figuratives, reproduites dans All One Horse, n'ont pas de légende ; toutefois, chacun des textes en prose possède un titre, que l'interprète est également tenté d'associer à l'aquarelle qui, souvent, lui fait face. Dans certains cas, le titre du texte en prose (qui est, à chaque fois, la reprise de son explicit) correspond assez bien à l'image située en regard.

Toutefois, les aquarelles des pages 53 et 109 fonctionnent suivant le même principe de «discrépance» que les trois toiles de Magritte nous ont permis d'évoquer. La toile reproduite en page 53 représente un homme en train de nager les yeux fermés ; la moitié supérieure représente une montagne de couleur ocre; sur ses flancs sont plantées des lettres géantes, qui forment le syntagme "mot». Chacune des trois occurrences (ou apparitions ?) du syntagme est associée à un pinceau ; un caméléon est accroché à la lettre $\mathrm{M} \mathrm{du}$ " mot » central. La présence du syntagme français « mot » n'a rien qui doive surprendre: le livre ne cesse d'insister sur la notion biblique de Verbe ${ }^{9}$ (en anglais: "Word», traduction possible du français «mot »). Le texte dans lequel cette aquarelle s'insère s'intitule d'ailleurs « The Body Quivers with the Word » (51-55). 

de « Word » dans le reste du recueil invite à la méfiance. De surcroît, comme le souligne Sandra Saayman, le syntagme "mot " signifie "phalène » en afrikaans («moth» en anglais). Par ce jeu sur les trois principales langues de référence de l'auteur semble s'affirmer l'idée d'une " discrépance " généralisée des motifs et des significations dans All One Horse. À cela s'ajoute une dimension intratextuelle essentielle, dans la mesure où la figuration du doublon «mot/word » joue un rôle primordial dans plusieurs textes de All One Horse : au jeu plurilinguistique ponctuel usant du sens de « mot » en afrikaans s'ajoute la représentation visuelle des initiales inversées $\mathrm{M}$ et $\mathrm{W}^{10}$. Bref: tout en ayant une multitude de signifiés linguistiques, le motif pictural composant le syntagme MOT n'a pas de référent clairement lisible ou définissable. quatre figures représentées (Victor Hugo nu, un singe au sexe en forme de cœur, une tête coupée clouée au sol et un boxeur noir amputé de ses deux jambes), le boxeur attire l'attention en raison des lettres inscrites sur ses vêtements : "oite» sur le gant protégeant la main droite ; "gauc » sur le gant protégeant la main gauche ; « haut » sur le casque ; «basin » sur le short. Si les trois premiers mots sont facilement identifiables en français, le quatrième est un substantif anglais sans pertinence descriptive ici, mais qui commence par les trois lettres formant l'adjectif antonyme de « haut » en français, et qui figure sur tous les colis confiés à des entreprises de déménagement en France (haut / bas). Bien que le syntagme "basin » soit assimilable, à une lettre près, au français «bassin », substantif descriptif superposé à son référent (le bas-ventre du boxeur) dans la toile, il n'en demeure pas moins «discrépant »: la polysémie contextuelle du terme ne permet nullement d'éclairer la réification du boxeur, réduit par les signes graphiques à l'état de colis endommagé.

19 La «discrépance " entre en jeu en raison des tentatives d'interprétation du lecteur, comme dans la mise en miroir de l'aquarelle de la page 116 et d'une description de lieu à la fois comparable et radicalement autre ${ }^{11}$.

Les descriptions elles-mêmes donnent la pleine mesure de cette impossibilité de l'écriture à imaginer, à mettre en images, à suggérer une ou plusieurs image(s) :

It should be pointed out that the place is nondescript, neither big nor cramped nor frankly evil - why should it be? - with some smoke adrift near the ceiling and drinks to be had in the sad foyer. A black cape of shiny hair throws a shadow over the glitter and glance in the lady's eyes. ("The Thieves and the Word », 41-42)

Seule la dernière phrase peut prétendre à un quelconque effet de description; toutefois, la présence d'énoncés poétiques (notamment la double allitération en [sh] et [gl]) contredit cet impératif de transparence, tout en soulignant l'ambivalence de l'expression "black cape", métaphore pour le moins opaque. Pour le reste, l'emploi même de l'adjectif «nondescript» (qui n'a pas d'équivalent strict en français) en dit assez sur la négativité qui sous-tend la relation entre texte et image: le rejet de deux adjectifs qualificatifs antithétiques («big/cramped»), puis d'un adjectif à connotation morale mis sur un même plan syntaxique ( evil») est certes producteur de sens, mais en creux. Seules deux activités humaines ordinaires («smoke», «drink») semblent dessiner plus précisément les contours ou la fonction du lieu en question, mais l'alliance d'un substantif concret et d'un adjectif moral ("sad foyer ») en fin de phrase conforte les ambivalences du passage. Ici, la signifiance va à l'encontre de l'impératif de référence inscrit (en théorie) dans tout énoncé descriptif. 
22 Par ailleurs, la « littérarité » des proses poétiques de Breyten Breytenbach a un effet général de perturbation sémantique, d'affolement du sens. Dans la mesure où le référent des «descriptions» est insaisissable, est-il encore possible de parler de texte « descriptif »? « Certains textes, bien évidemment, jouent à effacer systématiquement les frontières entre texte narratif et texte descriptif, entre texte pratique et textes fictifsmythiques (textes ne relevant pas de la distinction vrai-faux) $»^{12}$. Cette remarque de Philippe Hamon vaut particulièrement pour le premier paragraphe de « Remnants of my Story » :

We live deep in the country where it takes a long time to fashion a phrase to perfection. It rains incessantly, strands of hair cling to our foreheads, our hands are painted with water, our legs are browned to the thighs with mud. The trees submit to the ongoing gossiping of uncountable wet tongues. ("Remnants of my Story ", 29)

La qualité principale de cet incipit, c'est l'indistinct, l'indéterminable (" uncountable»). Dans la dernière phrase de la citation, par exemple, le lien logique entre les trois référents principaux (arbres-potins-langues) ne relève nullement $\mathrm{du}$ "texte pratique "; la phrase mêle savamment termes narratifs («submit») et descriptifs ( wet»), sans oublier le terme central ("gossiping»), fondamentalement hybride. De plus, la description de la contrée, du pays, n'a rien de physique ou de géographique ; il n'y est question que des qualités verbales des habitants. La description des mains ruisselant d'eau use d'une métaphore picturale (« painted with water ») qui en opacifie la référence. Ce paragraphe ne saurait même servir d'«illustration » de l'aquarelle qui lui fait face : seule l'expression "browned to the thighs with mud » rencontre un écho dans la matité des peaux du couple enlacé sur la table, ce qui constitue un lien fort fragmentaire... et, de fait, le titre de la nouvelle ne met-il pas en avant cette notion de fragment, de bribe (" remnants ») ? Faut-il chercher les bribes de l'histoire (" of my story ») dans les motifs picturaux? C'est peu probable.

\section{Le dé-scriptif}

Il s'agit alors de concevoir le descriptif littéraire non plus sous son aspect scripturaire, comme inscription, mais comme pure écriture. Le descriptif devient alors dé-scriptif, n'est plus mise en images.

Ainsi, la description du feu dans « Dead at Last » opacifie plus qu'elle ne donne à voir :

It was night. And a fire came raucously loping down the hill, snipping and puffing tree and undergrowth in its way.

In no time fire entered the courtyard making an eery day of the surroundings by the fiery glow of its body, barging into the barn and then jumping right across onto the roof of the house. It tore through the tiles and started licking at the beams with any number of black tongues. Thieves are so bold they sometimes steal your roof tiles while you are sitting down at supper. Sparks made a spray of fireworks and cinders rained down. ("Dead at Last », 74)

La figure principale est la personnification, qui ne permet pas véritablement de visualiser l'incendie. Dans sa dimension mytho-poiétique, une telle description suppose, de la part $\mathrm{du}$ lecteur, une connaissance préalable du phénomène physique décrit. Métaphores personnifiantes ("snipping and puffing »), tautologies (" fiery glow »), resémantisation de clichés (« any number of black tongues »)... toutes ces figures s'inscrivent dans une stratégie d'opacification. S'il est possible de voir dans ce passage un exemple 
d'hypotypose, il me semble que cette figure rhétorique (d'ailleurs difficilement formalisable), qui est généralement censée " donner à voir ", donne toutefois plus à lire, plus à entendre, par une surenchère poétique qui n'a rien de l'image.

Il en est de même pour la vision de la femme dans le lit défait :

This space - in fact it was a bathroom - they never entered, they just looked in from the outside. On the unmade bed next to the bath, among the warm sheets and as though protected by two pillows, lay the loveliest female head you ever could expect to behold, carved from a nearly transparent rose-hued jade or alabaster.

( "Jade or Alabaster », 91)

Hormis la désignation précise, entre tirets, de la fonction de la pièce (salle de bains), rien n'est clair : pourquoi y a-t-il un lit près de la baignoire ? Les figures de style insistent une nouvelle fois sur l'impossibilité de toute représentation verbale, qu'il s'agisse de la comparaison hypothétique ("as though») ou du superlatif ("the loveliest female you ever could expect to behold»). Le seul motif descriptif cohérent (formes et couleurs) est emprunté au vocabulaire de la sculpture, et ne permet nullement au lecteur d'imaginer la femme : à l'hésitation entre deux pierres assez différentes, qui donne d'ailleurs son titre au texte, s'ajoute la mention énigmatique "nearly transparent ", qui ne correspond à aucune caractéristique physique répertoriée.

C'est bien l'un des paradoxes du descriptif dans All One Horse: pour échapper simultanément à la figuration et à l'écriture, Breytenbach recourt à des expressions et des motifs typiquement esthétiques.

She washes up her dinghy on the shore, she has no oars, she clings to the sides of the boat, the sea is insurrectional like a Japanese woodblock print depicting the engulfment of coastline and small human figures, waves sough and smooch on the shale of the beach, sprays give the air a glisten and a sparkle. Flotsam and jetsam. The dowdy dress clings to her shapeless body, grey locks plastered to her forehead, her eyes lie low and sullen in their sodden coffins. (" Another Woman? », 107)

Tout en décrivant à merveille, ce texte ne décrit rien. Outre la surcharge d'éléments picturaux, qui servent d'écran plus que d'éclaircissement, on note la rareté des adjectifs, le plus souvent dé-scriptifs, qui n'inscrivent, ne suscitent aucune image («shapeless»); enfin, les figures rhétoriques reposant sur une structure double ("shore/no oars"; «sough and smooch»; «flotsam and jetsam»; «sullen/sodden») ont un effet plus textuel que référentiel.

\section{L'ekphrasis ou le retour du descriptif ?}

31 Dans un ouvrage où alternent vingt-sept textes et vingt-sept aquarelles figuratives, 1 'ekphrasis trouve naturellement sa place. En effet, malgré tout ce qui vient d'être dit au sujet de la déconstruction des référentiels au moyen d'une écriture primordialement signifiante, certains passages entretiennent l'illusion d'un tableau. Dans le deuxième texte du recueil, par exemple, c'est un sujet de tableau presque académique - la procession princière - qui est mis en avant :

The princess comes by all in silver, rouged cheeks and glittering eyes, six ladies-inwaiting carrying the train of her dress. She must be wearing boots for the mud. I wave. She doesn't respond. Nobody acknowledges me any more. Knights and hunters gallop by, pull up their chargers sharply to tumble over the necks of the steeds and perform somersaults on the ground. They slap their thighs with gloved 
hands. The street is lined with people bearing spluttering torches. («The Bobbing

Lights of the Harbour », 17) paragraphe de « No Longer». Le recours aux phrases nominales, ainsi qu'aux adjectifs précis en grand nombre, témoigne de cette résurgence du descriptif au sens classique du terme. D'ailleurs, il s'agit presque d'une fiche d'identification telle qu'elle pourrait être fournie par les renseignements généraux, à cette différence près que la description du pendu prend une forme passablement ironique :

Then, in the seventh paragraph, there is the man.

Name: Wormfood, though some know him as Bird-dream. Appearance: Hair like wool which has gathered dust. One cheek curving carved from the sun. Dried blood from one ear. The weal on the neck still fresh, angry, red. Lips drawn back so that the blue tongue protrudes. Eyelids half closed but eyes are looking at the back of the mind. Throat swollen with silence, stuck in the gullet a raucous word as purple as congealed blood.

Soiled collar. Cheap jacket. Nothing in the pockets except an ear of paper with the words: I am your brother.

Pants, a frayed hem. Underpants stained by ejaculated seed, traces of excretion. No socks. One shoe missing.

Body smooth, shoulders drooped, bell slumped, sphincter relaxed, veins on hands swollen, feet loose at ankles, toes with corns, some dirt between seven toes, about seventeen inches between black toes and floor. Purpose: truth. Truth: no longer. (« No Longer », 114-115) ${ }^{13}$

L'« effet de liste », pour reprendre l'expression de Philippe Hamon, est certes ekphrastique ; elle sert de point de référentialité, de point d'ancrage ou d'accroche d'une image. Cependant, les marges de ce portrait dénoncent le contrat. Les deux dernières rubriques échappent à la description physique conventionnelle.

La première phrase de la citation, quant à elle, souligne la nature foncièrement autoréféréntielle de ce passage : en effet, « No Longer » relate le contenu d'un texte fictif écrit par le dénommé Ka'afir et pourvu d'un double titre (The First Book of Slow Gestures ou The Papers of Guilt); or, la mention du « septième paragraphe » renforce la confusion entre le livre de Ka'afir et « No Longer », puisqu'il s'agit bel et bien du septième paragraphe que le lecteur a sous les yeux...!

36 La taxinomie, forme particulière de l'ekphrasis et de la référentialité, est ainsi happée par un co-texte hyper-formel qui la dénonce comme une illusion. Cette oscillation de la description littéraire entre opacité et transparence est d'ailleurs analysée par Philippe Hamon :

Effet de liste, effet de synonymie, ou effet de particularisation croissante se combinent alors, même s'il se peut que la référence soit compromise [...], même s'il se peut que le jeu sur le signifiant soit le principe organisateur et homogénéisateur 
[...] pour construire à la fois un groupe rythmique syncopé et, paradoxalement, une amplificatio textuelle combinée avec une restriction croissante d'extension et de compréhension de l'objet décrit. ${ }^{14}$ descriptif en prise avec un référent déterminable et un type de dé-scriptif aux prises avec la référence ; le descriptif qui élucide ou éclaire, et le descriptif qui opacifie, qui ne sert qu'à noircir... Bien entendu, ces deux formes sont des pôles, entre lesquels une infinie palette peut se développer. Il me semble que le descriptif mis en œuvre dans All One Horse. Fictions and Images relève assez nettement de la seconde catégorie, sans que je puisse pour autant prétendre avoir élaboré une théorie totalisante et définitive du descriptif : à peine ai-je esquissé un parcours fragmentaire et provisoire autour du descriptif dans certains textes de Breyten Breytenbach.

\section{NOTES}

1. London: Faber and Faber, 1990. Toutes les références renvoient à cette édition.

2. Et encore... N'est-il pas possible de concevoir un tableau comme fiction, en ce qu'il procède d'un fingere, de même que le terme d'« image » reste applicable au poème en prose ?

3. Breyten Breytenbach, Hart-Lam, Emmarentia: Taurus, 1991, 72.

4. Breyten Breytenbach, Painting the Eye, Cape Town: David Philip, 1993, 67.

5. Marilet Sienaert, «Ut pictura poesis? A Trangressive Reading of Breytenbach's Poetry and Painting », in Current Writing. vol. 8, $\mathrm{n}^{\circ} 1,1996,109$.

6. Sur la tentation du labyrinthe dans l'œuvre de Breytenbach, voir Sandra Saayman, Texte et image: la littérature de prison de Breyten Breytenbach, Thèse soutenue le 10 octobre 2003 à l'Université de Poitiers, sous la direction de Liliane Louvel. 
7. Ces deux tableaux, peu connus, sont reproduits dans le catalogue d'exposition dirigé par Margit Rowell (ed.), Objects of Desire: The Modern Still Life, New York: The Museum of Modern Art, 1997.

8. Isidore Isou, Euvres de spectacle, Paris: Gallimard, 1964, passim. Il ne s'agit pas ici d'un anglicisme évitable, mais bien plutôt d'un latinisme qui a valeur, dans l'œuvre d'Isou, de concept : est « discrépante » toute scène théâtrale dans laquelle un personnage parle de quelque chose tout en proposant une suite d'actions dénuées de lien avec le propos de son monologue. Ainsi, gestes et paroles, langage verbal et langage corporel, s'inscrivent dans une totale dichotomie.

9. Voir notamment : «In the beginning there is the Word. (This is plagiarism.)» (11), mais aussi la prose poétique intitulée "The Thieves and the Word» (41-44).

10. Voir l'aquarelle de la page 40 , avec son double $\mathrm{W}$, mais aussi la description de la génisse : «a bloated cow bobs along for all the world a dislocated upside-down $M »(29)$.

11. Cet exemple a déjà fait l'objet d'une analyse poussée dans mon article, « Du pareil au même? Les aquarelles et les proses poétiques de All One Horse ", in G. Cingal (ed.), Arts et textes en Afrique, Paris : APELA, 2000, 26-41.

12. Philippe Hamon, Du Descriptif, Paris : Hatier, 1993, 95.

13. Je ne m'attarde pas ici sur les effets de miroir déformant induits par la présence, à la page précédente, d'une aquarelle représentant un pendu qui n'est autre qu'un autoportrait. Discrépance, encore et toujours : Breyten, pendu, n'est pas vêtu, n'a pas la langue pendante, etc. Le pendu de l'image n'a rien de commun avec le pendu du texte (Wormfood/ Bird-dream, double nom ironique), si ce n'est son statut de pendu. Dans des registres sémiotiques et sémantiques différents, texte et image signifient selon des modes divergents, et ne peuvent par conséquent être réduits à un unique (et illusoire) référent.

14. Hamon, Du Descriptif, op. cit., 67.

15. Titre d'un des derniers chapitres des Misérables.

\section{INDEX}

oeuvrecitee All One Horse. Fictions and Images 